\title{
Five-Year Survival After Surgical Treatment for Kidney Cancer
}

\section{A Population-Based Competing Risk Analysis}

\author{
John M. Hollingsworth, MD ${ }^{1}$ \\ David C. Miller, MD, $\mathrm{MPH}^{2}$ \\ Stephanie Daignault, $\mathrm{ms}^{1}$ \\ Brent K. Hollenbeck, MD, ms ${ }^{1}$ \\ ${ }^{1}$ Department of Urology, University of Michigan, \\ Ann Arbor, Michigan. \\ ${ }^{2}$ Department of Urology, University of California \\ at Los Angeles, Los Angeles, California.
}

J.M. Hollingsworth is supported by NIH grant T-32-DK007758. Brent K. Hollenbeck is supported, in part, by the John and Suzanne Munn Endowed Research Fund of the University of Michigan Comprehensive Cancer Center.

Address for reprints: Brent K. Hollenbeck, MD, MS, 1500 E. Medical Center Dr., TC 3875-0330 Ann Arbor, Ml 48109-0330; Fax: (734) 936-9127; E-mail: bhollen@umich.edu

Received November 9, 2006; revision received January 14, 2007; accepted January 17, 2007.
BACKGROUND. Kidney cancer's rising incidence is largely attributable to the increased detection of small renal masses. Although surgery rates have paralleled this incidence trend, mortality continues to rise, calling into question the necessity of surgery for all patients with renal masses. Using a population-based cohort, a competing risk analysis was performed to estimate patient survival after surgery for kidney cancer, as a function of patient age and tumor size at diagnosis.

METHODS. With data from the Surveillance, Epidemiology, and End Results Program (1983-2002), a cohort was assembled of 26,618 patients with surgically treated, local-regional kidney cancer. Patients were sorted into 20 age-tumor size categories and the numbers of patients that were alive, dead from kidney cancer, and dead from other causes were tabulated. Poisson regression models were fitted to obtain estimates of cancer-specific and competing-cause mortality.

RESULTS. Age-specific kidney cancer mortality was stable across all size strata but varied inversely with tumor size. Patients with the smallest tumors enjoyed the lowest cancer-specific mortality ( $5 \%$ for masses $\leq 4 \mathrm{~cm}$ ). Competing-cause mortality rose with increasing patient age. The estimated 5-year competing-cause mortality for elderly subjects ( $\geq 70$ years) was $28.2 \%$ (95\% confidence interval [CI]: $25.9 \%-30.8 \%)$, irrespective of tumor size.

CONCLUSIONS. Despite surgical therapy, competing-cause mortality for patients with renal masses rises with increasing patient age. After 5 years, one-third of elderly patients ( $\geq 70$ years) will die from other causes, suggesting the need for prospective studies to evaluate the role of active surveillance as an initial therapeutic approach for some small renal masses. Cancer 2007;109:1763-8. (C) 2007 American Cancer Society.

KEYWORDS: mortality, kidney neoplasms, surgery, SEER program.

$T$ he rising incidence of kidney cancer demonstrated over the past 2 decades $^{1-4}$ is largely attributable to an increase in the number of small renal masses. ${ }^{5}$ Reflecting the current treatment paradigm, ${ }^{6}$ the rising incidence has been paralleled by greater use of nephrectomy. ${ }^{5}$ Despite these trends, kidney cancer mortality rates have continued their monotonous rise. ${ }^{5}$ This apparent disconnect-between increasing treatment and increasing mortality-raises questions regarding the necessity of surgery for all patients with small kidney tumors.

There is growing evidence to suggest that the anticipated survival benefit derived from the surgery for suspicious renal masses is nonuniform. To begin, kidney cancer is now recognized as a heterogeneous disease, whose histopathologic subtypes have variable natural 
histories. ${ }^{7,8}$ For this reason, it is plausible that a proportion of newly diagnosed renal masses represent an indolent form of cancer, which may not merit surgical removal. Given the low metastatic potential of some nonclear-cell variants ${ }^{7}$ and the slow growth rate observed for many incidentally detected lesions, ${ }^{9}$ it is also conceivable that age-related competing-cause mortality might dampen the benefits of surgery for some older patients with small renal masses. Because the morbidity and mortality of nephrectomy are nontrivial, ${ }^{10}$ clinicians must be able to better risk-stratify kidney cancer patients such that those who stand to gain the most from surgical intervention receive it. To better inform the clinician counseling a patient with a newly diagnosed renal mass, we estimated patient survival after surgery for kidney cancer by performing a competing risk analysis on a population-based cohort with local-regional disease.

\section{MATERIALS AND METHODS}

We used data from 9 Surveillance, Epidemiology, and End Results (SEER) registries (San Francisco-Oakland, Connecticut, Metropolitan Detroit, Hawaii, Iowa, New Mexico, Seattle-Puget Sound, Utah, Metropolitan Atlanta, San Jose-Monterey, Los Angeles, Alaska) to identify incident cases of kidney cancer based on International Classification of Disease for Oncology, Second Edition ${ }^{11}$ site code C64.9; histology codes 8032, 8041, 8140, 8240, 8260, 8270, 8290, 8310, 8312, 8317, 8318, 8319, 8320, 8960, 8963, and 8966; and behavior codes 2 and 3 [for adenocarcinoma in situ and adenocarcinoma not otherwise specified (NOS), respectively] occurring between 1983 and 2002.

Our study cohort was limited to those patients with local-regional disease (according to SEER's 'best' staging system) who were treated with definitive surgical therapy (ie, radical or partial nephrectomy and locally ablative therapies). We ascertained the receipt of surgical therapy based on SEER's site-specific surgery codes 10, 20, 30, 40, 50, 60, 70, and 90 (1983 to 1997). For the years 1998 to 2002, SEER added codes for local tumor destruction (12,13, and 14), local tumor excision $(22,23,24,25,26$, and 27), and nephrectomy with ureterectomy (80). The preceding list was appended with these codes. Using this approach, we identified 27,968 patients with surgically treated, local-regional kidney cancer. We excluded 1350 patients $(4.8 \%)$ with missing data for tumor size, yielding a final study sample of 26,618 patients.

Demographic data, including age at diagnosis, year of diagnosis, race, marital status, length of follow-up, surgery type, and vital status at last contact, were extracted. With the exception of patient age trea- ted as a continuous variable, we treated each of these factors as a categorical variable. Next, for analytic purposes we assigned patients to 1 of 20 mutually exclusive and exhaustive tumor size-age strata. Specifically, we first assigned patients to 1 of 4 distinct tumor size categories $(<2,2-4,>4-7$, and $>7 \mathrm{~cm}$ in size); within each tumor size stratum we then defined 5 age categories $(<50,50-59,60-69,70-79$, and $\geq 80$ years).

\section{Statistical Methods}

The primary focus of this study was to estimate the probability of mortality from kidney cancer or other competing causes after surgical intervention, given a patient's age and tumor size at diagnosis. Therefore, we tabulated the numbers of patients with each of the 3 outcomes of interest (alive, dead from kidney cancer, and dead from other causes) for each of our 20 age-tumor size combinations.

We then used separate Poisson regression models to obtain estimates of the mortality rates from kidney cancer and other competing medical conditions. Finally, we applied the fitted rates of kidney cancer death and other-cause death to the proportion of patients still alive at the beginning of each successive 1-year follow-up interval. This provided us with estimates of the proportion of patients who died from kidney cancer and other competing causes, or who were still alive 5 years after diagnosis. These estimates were then plotted for each age-tumor size stratum. All statistical tests were 2-tailed and performed at a significance level of .05 using the SAS system (v. 9.1, SAS Institute, Cary, NC). Institutional Review Board approval was waived for this study.

\section{RESULTS}

The characteristics of the study cohort $(n=26,618)$ are described in Table 1. The typical study subject was a married, white male over age 60 . Nearly all (92\%) underwent a radical nephrectomy for management of their local-regional disease. Five years of follow-up data were available for $46 \%$, and $37 \%$ died from kidney cancer or other causes over the study interval.

Table 2 presents the distribution of death from kidney cancer or competing causes stratified by tumor size and age at diagnosis. Among patients with small $(\leq 4 \mathrm{~cm})$ surgically treated lesions, only $5 \%$ died from kidney cancer within 5 years. In contrast, even with surgery $27 \%$ of patients with the largest $(>7 \mathrm{~cm})$ lesions died from their disease.

Figure 1 depicts the smoothed, model-derived, 5 -year cumulative mortality estimates. Age-specific mortality from kidney cancer showed relative stability 
TABLE 1

Characteristics of Patients With Treated Local-Regional Kidney Cancer (1983-2002)

\begin{tabular}{lc}
\hline & $\mathbf{N}=\mathbf{2 6 , 6 1 8}$ \\
Characteristic & No. (\%) \\
\hline SEER site & \\
San Francisco & $3394(13)$ \\
Connecticut & $4136(15)$ \\
Metro Detroit & $5439(20)$ \\
Hawaii & $964(4)$ \\
Iowa & $3816(14)$ \\
New Mexico & $1575(6)$ \\
Seattle & $4031(15)$ \\
Utah & $1224(5)$ \\
Atlanta & $2039(8)$ \\
Age at diagnosis \pm standard deviation, y & $60.8 \pm 15.4$ \\
Median year of diagnosis [range] & $1995[1983-2002]$ \\
Race/ethnicity & \\
White & $21,390(81)$ \\
Hispanic & $1291(5)$ \\
Black & $2518(9)$ \\
Other & $1337(5)$ \\
Women & $10,031(38)$ \\
Marital status & \\
Single & $2964(11)$ \\
Married & $17,270(65)$ \\
Separated/divorced & $1951(7)$ \\
Widowed & $3181(12)$ \\
Follow-up & \\
Until death & \\
Alive at 1 y & $9966(37)$ \\
Alive at 3 y & $22,708(85)$ \\
Alive at 5 y & $16,631(62)$ \\
Treatment within 6 mo & $12,295(46)$ \\
Total nephrectomy & \\
Partial nephrectomy & $24,502(92)$ \\
Other surgery* & $2013(7.5)$ \\
Vital status at last contact & $92(0.5)$ \\
Alive & $16,652(63)$ \\
Deceased due to renal cell & $4006(15)$ \\
Deceased due to other causes & $5960(22)$ \\
\hline &
\end{tabular}

* Examples include cryosurgery and laser ablation.

within each of our 4 size strata, eg, for tumors $2-4 \mathrm{~cm}$ in size: those $<50$ years had a 5 -year mortality rate of $2.8 \%$ (95\% confidence interval [CI]: $2.4 \%-3.2 \%$ ); those $50-59$ years had a 5 -year mortality rate of $4.6 \%(95 \%$ CI: $4.1 \%-5.3 \%)$; those $60-69$ years had a 5 -year mortality rate of $5.5 \%$ (95\% CI: $4.8 \%-6.2 \%$ ); those $70-79$ years had a 5 -year mortality rate of $6.3 \%$ (95\% CI: $5.5 \%-7.1 \%$ ); and those $\geq 80$ years had a 5 -year mortality rate of $7.5 \%$ (95\% CI: $6.4 \%-8.8 \%$ ). However, cancer-specific mortality varied inversely with tumor size, such that patients with the smallest cancers had the lowest predicted cancer-specific mortality $(5.3 \%$, $95 \%$ CI: $4.6 \%-6.1 \%$, for those with tumors $\leq 4 \mathrm{~cm}$ vs $18.1 \%$, 95\% CI: $16.4 \%-20.0 \%$, for those with tumors $>4 \mathrm{~cm}$ ). In contrast, competing-cause mortality rose
TABLE 2

Five-Year Outcomes for Those Patients With Treated Local-Regional Kidney Cancer According to Tumor Size

\begin{tabular}{|c|c|c|c|c|c|c|}
\hline & \multicolumn{6}{|c|}{ Age at diagnosis } \\
\hline & $<50$ & $50-59$ & $60-69$ & $70-79$ & $\geq 80$ & $\begin{array}{l}\text { Total no. of } \\
\text { patients (\%) }\end{array}$ \\
\hline & & & & $<2 \mathrm{~cm}$ & & \\
\hline \multicolumn{7}{|c|}{ Sample size $(\mathrm{n}=1291)$} \\
\hline \multicolumn{7}{|c|}{ No. of patients deceased due to } \\
\hline RCC & 14 & 18 & 24 & 20 & 3 & 79 (6) \\
\hline Other causes & 27 & 27 & 81 & 101 & 17 & $253(20)$ \\
\hline No. of patients alive & 278 & 229 & 242 & 179 & 31 & $959(74)$ \\
\hline \multicolumn{7}{|c|}{$2-4 \mathrm{~cm}$} \\
\hline \multicolumn{7}{|c|}{ Sample size $(\mathrm{n}=8278)$} \\
\hline \multicolumn{7}{|c|}{ No. of patients deceased due to } \\
\hline $\mathrm{RCC}$ & 31 & 75 & 141 & 147 & 52 & $446(5)$ \\
\hline Other causes & 113 & 243 & 626 & 749 & 279 & $2010(25)$ \\
\hline No. of patients alive & 1286 & 1365 & 1615 & 1294 & 262 & $5822(70)$ \\
\hline \multicolumn{7}{|c|}{$>4-7 \mathrm{~cm}$} \\
\hline \multicolumn{7}{|c|}{ Sample size $(\mathrm{n}=9105)$} \\
\hline \multicolumn{7}{|c|}{ No. of patients deceased due to } \\
\hline $\mathrm{RCC}$ & 152 & 249 & 402 & 365 & 121 & $1289(14)$ \\
\hline Other causes & 97 & 282 & 659 & 867 & 313 & $2218(24)$ \\
\hline No. of patients alive & 1312 & 1449 & 1542 & 1041 & 254 & $5598(62)$ \\
\hline \multicolumn{7}{|c|}{$>7 \mathrm{~cm}$} \\
\hline \multicolumn{7}{|c|}{ Sample size $(\mathrm{n}=7944)$} \\
\hline \multicolumn{7}{|c|}{ No. of patients deceased due to } \\
\hline $\mathrm{RCC}$ & 348 & 531 & 681 & 506 & 126 & $2192(27)$ \\
\hline Other causes & 107 & 211 & 438 & 522 & 201 & $1479(19)$ \\
\hline No. of patients alive & 1412 & 1065 & 994 & 650 & 152 & $4273(54)$ \\
\hline \multicolumn{7}{|c|}{ All tumors } \\
\hline \multicolumn{7}{|c|}{ Sample size $(\mathrm{n}=26,618)$} \\
\hline \multicolumn{7}{|c|}{ No. of patients deceased due to } \\
\hline $\mathrm{RCC}$ & 545 & 873 & 1248 & 1038 & 302 & $4006(15)$ \\
\hline Other causes & 344 & 763 & 1804 & 2239 & 810 & $5960(22)$ \\
\hline No. of patients alive & 4288 & 4108 & 4393 & 3164 & 699 & $16,652(63)$ \\
\hline
\end{tabular}

with increasing patient age. For instance, the predicted, 5-year competing risk mortality for those aged 70 years and older was $28.2 \%$ (95\% CI: $25.9 \%-30.8 \%$ ), irrespective of tumor size.

\section{DISCUSSION}

The relative benefit of definitive surgical therapy for small renal masses $(\leq 4 \mathrm{~cm})$ is partially mitigated by competing causes of mortality in older patients. For example, nearly one-third of patients aged 70 years and older will die from unrelated comorbid disease within 5 years of curative surgery for their kidney cancer. Prior work has demonstrated a rise in the incidence of these small, presumably curable, renal masses that has been paralleled by increases in surgical ther- 


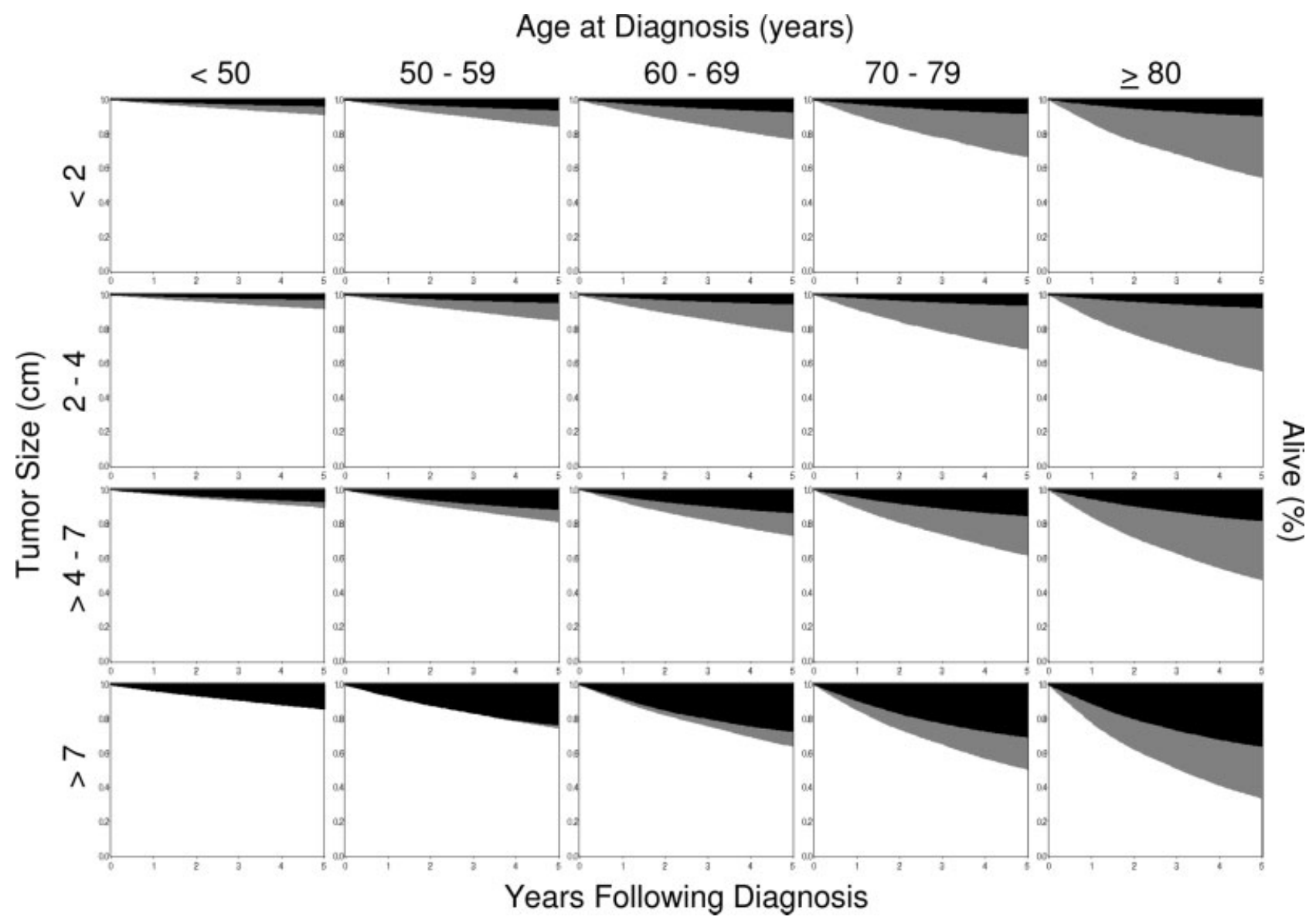

FIGURE 1. Five-year survival after surgical treatment for kidney cancer. Cumulative mortality for kidney cancer and all causes up to 5 years after diagnosis, after definitive surgical therapy. White indicates survival; gray indicates nonkidney cancer mortality; black indicates kidney cancer mortality.

apy. ${ }^{5}$ However, despite these 2 epidemiological trends, kidney cancer mortality has continued its gradual climb, ${ }^{5}$ suggesting that the current treatment algorithm be revisited.

This long-standing treatment paradigm ${ }^{12}$ for patients with a suspicious renal mass generally involves expedient surgical excision shortly after diagnosis. The reasons for this are 2-fold. First, systemic medical therapy and radiation regimens for kidney cancer have been shown to be generally ineffective. ${ }^{13-15} \mathrm{Ac}$ cordingly, surgery represents the best opportunity for curative intervention. Second, patients with larger, more advanced cancers face a dismal prognosis; ${ }^{16}$ therefore, surgery early in the disease is believed to improve their chance of survival.

With respect to this treatment paradigm, an important underlying assumption, one not supported by several case series, ${ }^{17-22}$ is that all small renal masses have the universal capacity to grow and metastasize. This assumption, however, has several possible flaws. First, kidney cancer is not a single entity; rather, it is a family of neoplasms with observed variants that have distinct cytogenetic defects and histopathologic features. ${ }^{8}$ For example, nonclear-cell sporadic types (eg, chromophobe renal cell carcinoma) often have an indolent course and a much lower metastatic potential compared with the clear-cell variety. ${ }^{7}$ In addition to the heterogeneous behavior of the various histologies, kidney cancers may exhibit variable growth according to their size. Indeed, small, suspicious tumors tend to grow at a relatively slow pace $(0.28 \mathrm{~cm} /$ year $) .{ }^{9}$ The indolent nature of these lesions is further bolstered by autopsy data, revealing that many incidentally found, small renal masses have less malignant potential than clinically detected tumors. ${ }^{23}$ Given these data, it is possible that a proportion of newly diagnosed renal masses may not merit immediate surgical removal.

Nonetheless, in the absence of robust data regarding the natural history of these small renal masses, it is impossible to truly understand their nature. Indeed, all large renal masses, which these data and that of many case series ${ }^{24-26}$ suggest are clearly lethal even after treatment, were small at one time or another. However, the current study suggests that the relative 
benefits of surgical treatment are lowest among older patients (eg, age $\geq 70$ years) with renal masses $\leq 4 \mathrm{~cm}$ in size. Thus, in this population, especially where there is significant concurrent comorbidity, a period of active surveillance may be warranted.

For the most part, contemporary surveillance protocols for small renal masses have been limited to patients with substantial medical comorbidity, generally thought to be too infirm to tolerate extirpative procedures. However, as safety of active surveillance increases through improvements in imaging and biopsy techniques, its role will likely continue to broaden. Until this time, these data provide insight into current clinical practice. Despite the fact that partial nephrectomy offers equivalent local tumor control to its radical counterpart, ${ }^{27,28}$ national trends illustrate that the uptake of nephron-sparing surgery for small $(\leq 4 \mathrm{~cm})$ renal masses has been slow. ${ }^{29}$ These utilization trends are troubling when considered in the context of recent findings that show radical nephrectomy to be a significant risk factor for the development of chronic kidney disease. ${ }^{30}$ The potential consequences of kidney disease, coupled with increasing competing-cause mortality with age, encourage a rethinking of the treatment algorithm for small renal masses.

Our findings must be considered in the context of several limitations. SEER does not collect data on comorbid status, which worsens with age and affects patient survival. However, we would argue that casemix adjustment in the setting of our competing risk analysis would have been inappropriate, as our intent was to estimate a patient's probability of cancer-specific vs competing-cause mortality-a probability intimately related to a patient's comorbidities. In addition, we determined the underlying cause of a patient's death using SEER's cause-of-death item. The validity of this construct, which is based on death certificate reporting, has been called into question in several settings, specifically as it relates to issues of race and socioeconomics. ${ }^{31-33}$ However, this approach of measuring cause-of-death has compared favorably with that obtained from autopsy among patients with cancer diagnoses when tested empircally. ${ }^{34}$ Thus, although we cannot exclude residual bias related to coding, these data support the use of this construct in the current context.

\section{Conclusions}

In summary, our study demonstrates that a significant proportion of kidney cancer patients will die within 5 years of diagnosis despite definitive surgical therapy, given the relation between competing-cause mortality and increasing age. While we are not encouraging an abrupt departure from the current treatment paradigm, our data do prompt reflection on contemporary practice patterns for kidney cancer. Further, our data suggest the need for prospective studies to evaluate the role of active surveillance as an initial therapeutic approach for some small renal masses.

\section{REFERENCES}

1. Chow WH, Devesa SS, Warren JL, et al. Rising incidence of renal cell cancer in the United States. JAMA. 1999;281: 1628-1631.

2. Hock LM, Lynch J, Balaji KC. Increasing incidence of all stages of kidney cancer in the last 2 decades in the United States: an analysis of Surveillance, Epidemiology and End Results program data. J Urol. 2002;167:57-60.

3. Ries LAG, Eisner MP, Kosary CL, et al. SEER Cancer Statistics Review, 1975-2002. Based on November 2004 SEER data. National Cancer Institute. Bethesda, MD, 2005. Available at URL: http://seer.cancer.gov/csr/1975_2002

4. Vaishampayan UN, Do H, Hussain M, et al. Racial disparity in incidence patterns and outcome of kidney cancer. Urology. 2003;62:1012-1017.

5. Hollingsworth JM, Miller DC, Daignault S, et al. Rising incidence of small renal masses: a need to reassess treatment effect. J Natl Canc Inst. 2006;98:1331-1334.

6. Kidney Cancer Clinical Practice Guidelines in Oncology (Version 2.2006). (c) 2006 National Comprehensive Cancer Network, Inc. Available at URL: http://www.nccn.org Accessed on September 23, 2006.

7. Kattan MW, Reuter V, Motzer RJ, et al. A postoperative prognostic nomogram for renal cell carcinoma. J Urol. 2001; 166:63-67.

8. Zambrano NR, Lubensky IA, Merino MJ, et al. Histopathology and molecular genetics of renal tumors toward unification of a classification system. J Urol. 1999;162:1246-1258.

9. Chawla SN, Crispen PL, Hanlon AL, et al. The natural history of observed enhancing renal masses: meta-analysis and review of the world literature. J Urol. 2006;175:425431.

10. Taub DA, Miller DC, Cowan JA, et al. Impact of surgical volume on mortality and length of stay after nephrectomy. Urology. 2004;63:862-867.

11. Percy C, Van Holten V, Muir C. International Classification of Diseases for Oncology. 2nd ed. Geneva: World Health Organization; 1990.

12. Robson CJ. Radical nephrectomy for renal cell carcinoma. J Urol. 1963;89:37-41.

13. Yagoda A, bi-Rached B, Petrylak D. Chemotherapy for advanced renal-cell carcinoma: 1983-1993. Semin Oncol. 1995;22:42-60.

14. Onufrey V, Mohiuddin M. Radiation therapy in the treatment of metastatic renal cell carcinoma. Int J Radiat Oncol Biol Phys. 1985;11:2007-2009.

15. Bukowski RM. Chemoimmunotherapy of metastatic renal cell carcinoma. Cancer Invest. 1999;17:460-461.

16. Figlin RA. Renal cell carcinoma: management of advanced disease. J Urol. 1999;161:381-386.

17. Bosniak MA, Birnbaum BA, Krinsky GA, et al. Small renal parenchymal neoplasms: further observations on growth. Radiology. 1995;197:589-597.

18. Kassouf W, Aprikian AG, Laplante M, et al. Natural history of renal masses followed expectantly. J Urol. 2004;171:111113. 
19. Kato M, Suzuki T, Suzuki Y, et al. Natural history of small renal cell carcinoma: evaluation of growth rate, histological grade, cell proliferation and apoptosis. J Urol. 2004;172: 863-866.

20. Oda T, Miyao N, Takahashi A, et al. Growth rates of primary and metastatic lesions of renal cell carcinoma. Int $\mathrm{J}$ Urol. 2001;8:473-477.

21. Volpe A, Panzarella T, Rendon RA, et al. The natural history of incidentally detected small renal masses. Cancer. 2004;100:738-745.

22. Wehle MJ, Thiel DD, Petrou SP, et al. Conservative management of incidental contrast-enhancing renal masses as safe alternative to invasive therapy. Urology. 2004;64:49-52.

23. Kihira T, Shiraishi T, Yatani R, et al. Pathological features of renal cell carcinoma incidentally discovered at autopsy. Acta Pathol Jpn. 1991;41:680-684.

24. Gettman MT, Blute ML, Spotts B, et al. Pathologic staging of renal cell carcinoma: significance of tumor classification with the 1997 TNM staging system. Cancer. 2001;91:354-361.

25. Grignon DJ, Ayala AG, el-Naggar A, et al. Renal cell carcinoma. A clinicopathologic and DNA flow cytometric analysis of 103 cases. Cancer. 1989;64:2133-2140.

26. Hafez KS, Fergany AF, Novick AC. Nephron sparing surgery for localized renal cell carcinoma: impact of tumor size on patient survival, tumor recurrence and TNM staging. J Urol. 1999;162:1930-1933.
27. Lee CT, Katz J, Shi W, et al. Surgical management of renal tumors $4 \mathrm{~cm}$. or less in a contemporary cohort. J Urol. 2000;163:730-736.

28. Fergany AF, Hafez KS, Novick AC. Long-term results of nephron sparing surgery for localized renal cell carcinoma: 10-year followup. J Urol. 2000;163:442-445.

29. Miller DC, Hollingsworth JM, Hafez KS, et al. Partial nephrectomy for small renal masses: an emerging quality of care concern? J Urol. 2006;175:853-885.

30. Huang WC, Levey AS, Serio AM, et al. Chronic kidney disease after nephrectomy in patients with renal cortical tumours: a retrospective cohort study. Lancet Oncol. 2006;7: 735-740.

31. Pritt BS, Hardin NJ, Richmond JA, et al. Death certification errors at an academic institution. Arch Pathol Lab Med. 2005;129:1476-1479.

32. Schade WJ, Swanson GM. Comparison of death certificate occupation and industry data with lifetime occupational histories obtained by interview: variations in the accuracy of death certificate entires. Am J Ind Med. 1988;14:121-136.

33. Harwell TS, Hansen D, Moore KR, et al. Accuracy of race coding on American Indian death certificates, Montana 1996-1998. Public Health Rep. 2002;117:44-49.

34. Kircher T, Nelson J, Burdo H. The autopsy as a measure of accuracy of the death certificate. New Engl J Med. 1985;313: 1263-1269. 